Gut, 1983, 24, 206-212

\title{
Relapse of antibiotic associated colitis: endogenous persistence of Clostridium difficile during vancomycin therapy
}

\author{
B A J WALTERS, R ROBERTS, R STAFFORD, AND E SENEVIRATNE
}

From the Department of Microbiology, Repatriation General Hospital, Brisbane, Australia, and the Department of Medicine, University of Queensland, Queensland, Australia

SUMmary This study reports 24 patients with antibiotic associated colitis due to Clostridium difficile. Fifteen patients were treated with vancomycin due to the severity of the colitis and in eight of these a clinical relapse of the colitis occurred after vancomycin therapy was stopped. Bacteriological investigations of these patients indicated that $C$ difficile was able to persist in stool samples during vancomycin therapy in the absence of detectable cytotoxin. This was in contrast with the seven patients successfully treated with vancomycin without relapse, and those not treated with vancomycin where both stool cultures and cytotoxin assays became negative. These results suggest that patients being treated with vancomycin for antibiotic associated colitis due to $C$ difficile should have stool cultures done during and after treatment. Persistence of the organism in the absence of detectable cytotoxin may identify those patients who relapse and lead to either recommencement of vancomycin or alternative therapeutic approaches.

Oral vancomycin has been shown to be effective in the treatment colitis caused by $C$ difficile. ${ }^{1-3}$ In some patients relapses have been reported after vancomycin therapy was discontinued ${ }^{4-6}$ and alternative treatment has been used or considered. These include bacitracin orally, ${ }^{7}$ metronidazole, ${ }^{8}$ and the use of cholestyramine alone or in sequence with an antibiotic. ${ }^{9}$ Possible reasons for relapse after vancomycin treatment include a failure to totally eradicate the organism from the bowel, reinfection from a contaminated environment, or a combination of these. Resistance to vancomycin has not been described for $C$ difficile and strains isolated during relapse have all been sensitive. ${ }^{+6}$ Spore formation by $C$ difficile has been observed in the stool of animals being treated with vancomycin and these return to the vegetative form when vancomycin is terminated. ${ }^{10}$ Vancomycin is not active against bacterial spores. This provides one possible mechanism whereby $C$ difficile may persist in the presence of the high concentration of vancomycin achieved in the stool by standard oral doses. ${ }^{23}$ In vitro studies have shown that subinhibitory concen-

Address for correspondence and reprint requests: Dr E Seneviratne. Department of Microbiology. Repatriation General Hospital. Newdegate Street. Greenslopes. 4120 . Australia.

Received for publication 14 June 1982 trations of vancomycin can increase toxin production by $C$ difficile ${ }^{11}$ and normal gastrointestinal flora can inhibit its growth. ${ }^{12}$ Thus the time taken for some patients to re-establish their normal gastrointestinal flora after vancomycin therapy together with the persistence of $C$ difficile as spores may predispose them to relapses as toxin production recommences with falling vancomycin levels.

Reinfection from environmental sources has been suggested as another possible cause of relapse. ${ }^{413}$ Several studies have reported environmental contamination by $C$ difficile during and after the presence of patients known to be excreting the organism. ${ }^{14} 15$ These studies also found that $C$ difficile was able to persist in the environment for several months, thus providing an exogenous source of reinfection. There does not appear to be any predisposing factors that allow patients who relapse after vancomycin therapy to be identified. Bacteriological assessment of patients with antibiotic associated $C$ difficile colitis has usually been confined to the initial diagnosis and confirmation of the relapse. No studies have been concerned with bacteriological assessments during vancomycin therapy. This paper describes our observations on serial stool sampling of patients 
with antibiotic associated $C$ difficile colitis who were treated either with vancomycin or by stopping antibiotics.

\section{Methods}

\section{PATIENTS}

Initial stool specimens were submitted to the laboratory from inpatients and outpatients when the clinical history and/or sigmoidoscopy suggested antibiotic associated colitis. Diagnosis of antibiotic associated colitis was suspected when there was diarrhoea starting on or soon after a course of antibiotics, the absence of past or subsequent evidence of non-infective inflammatory bowel disease, the presence of stool leucocytes, and the absence of other enteric pathogens in stool culture. Confirmation of the diagnosis was indicated by the isolation of $C$ difficile in the absence of other enteric pathogens and the demonstration of a faecal cytotoxin neutralised by $C$ sordellii antitoxin. Patients treated with vancomycin were all inpatients and follow-up specimens were obtained while the patients were being treated and after discharge from hospital. Patients not treated had less severe symptoms at the time of submission of the first specimen and after discharge follow-up specimens were obtained from four out of nine patients. Patients with relapses of colitis had recurrence of diarrhoea and were documented by sigmoidoscopy, reisolation of $C$ difficile, detection of the cytotoxin, and the absence of other enteric pathogens. None of the patients had been on antimicrobial agents in the interval between the end of vancomycin therapy and the relapse. Other relevant clinical information appears in the tables.

\section{TECHNIQUES}

Stools collected from patients being investigated for antibiotic associated diarrhoea were assayed for the presence of cytotoxin neutralised by $C$ sordellii antitoxin in a W138 cell line. ${ }^{16}$ Cultural techniques included plating of the specimen, emulsified in reinforced clostridial broth RCB (Oxoid Ltd, London, England), on to cefoxitin, cycloserine fructose agar $\mathrm{CCFA}^{17}$ and a commercially available modification of this, $C$ difficile agar CDA (Oxoid Ltd, London, England). Approximately $2 \mathrm{ml}$ of the emulsified stool was inoculated directly into RCB containing $0.2 \% \mathrm{p}$ cresol. ${ }^{18}$ Subculture of this broth to selective media (CCFA, CDA) was routinely done at two and seven days. Cultures were incubated anaerobically at $37^{\circ} \mathrm{C}$ and colonies having the gross morphology of $C$ difficile were identified using Virginia Polytechnic Institute methods. ${ }^{19}$ Sensitivity of the isolates to vancomycin was determined in broth by standard methods. ${ }^{211}$ Toxin production by the isolates was determined on the supernates of $48 \mathrm{~h}$ cooked meat medium. Other bacterial pathogens including Salmonella, Shigella, Campylobacter and pathogenic Vibrio were excluded as well as parasites ova and cysts. All the cultures for bacterial pathogens were performed on stools within one hour of collection and cytotoxin assays were set up on the same day or on these specimens stored overnight at $-20^{\circ} \mathrm{C}$.

\section{Results}

The clinical and laboratory findings for the eight patients infected with $C$ difficile who were treated with vancomycin and had relapses are shown in Table 1 . The strains of $C$ difficile isolated from these patients at the time they were being treated with vancomycin were all toxigenic in the tissue culture assay although cytotoxin was not detected in serial spool specimens obtained from these patients at that time. The number of specimens obtained during therapy from each individual patient varied; two patients (CD and RF) were inpatients and specimens were obtained daily; two patients (FC and $\mathrm{GH}$ ) supplied four specimens and the remaining patients supplied two specimens each. All these specimens without exception were shown to contain $C$ difficile in the absence of detectable cytotoxin. Relapses occurred as early as three days (patient $\mathrm{CD}$ ) and as late as 28 days (patient $\mathrm{GH}$ ) after the cessation of vancomycin. In the eight patients who relapsed vancomycin retreatment resulted in a cure in six and $C$ difficile was not isolated nor the cytotoxin detected in serial stool specimens collected during the second treatment. With one patient in this group (patient $C D$ ) retreatment was not considered and in another (patient RF) retreatment did not cure the diarrhoea. These two patients continued to have diarrhoea and excrete the organism up to the time of their death because of unrelated causes.

In Table 2 are listed the patients treated with vancomycin who were cured without relapse. Serial stool specimens from these patients while being treated with vancomycin were both culture and cytotoxin negative for $C$ difficile. All the patients treated with vancomycin had severe colitis determined by sigmoidoscopy and in 14/15 there was evidence of a pseudomembrane. Initial vancomycin therapy was terminated for both groups when the clinical symptoms resolved although in four patients who had relapses (patients $\mathrm{GH}, \mathrm{AM}, \mathrm{CD}$, and RF) there was not a complete return to prediagnosis bowel frequency. Patients receiving no treatment (Table 3) had less severe symptoms and stool 


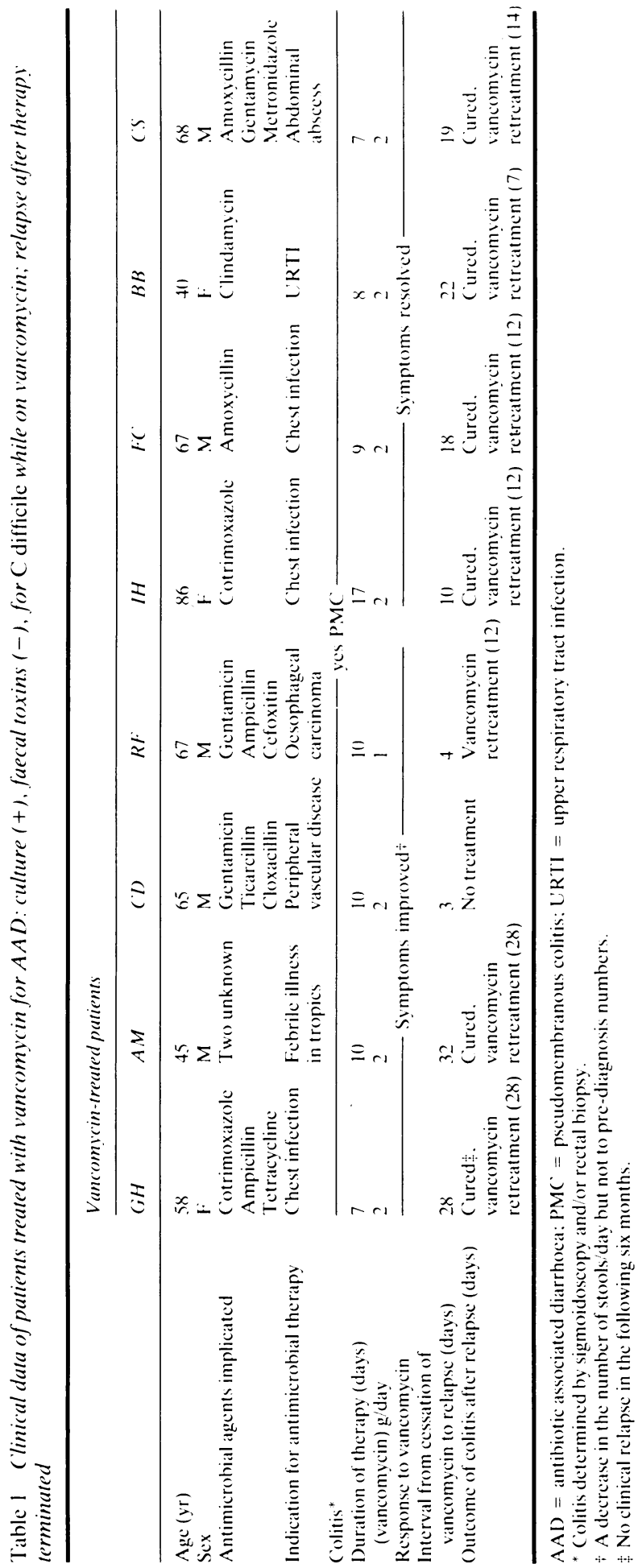



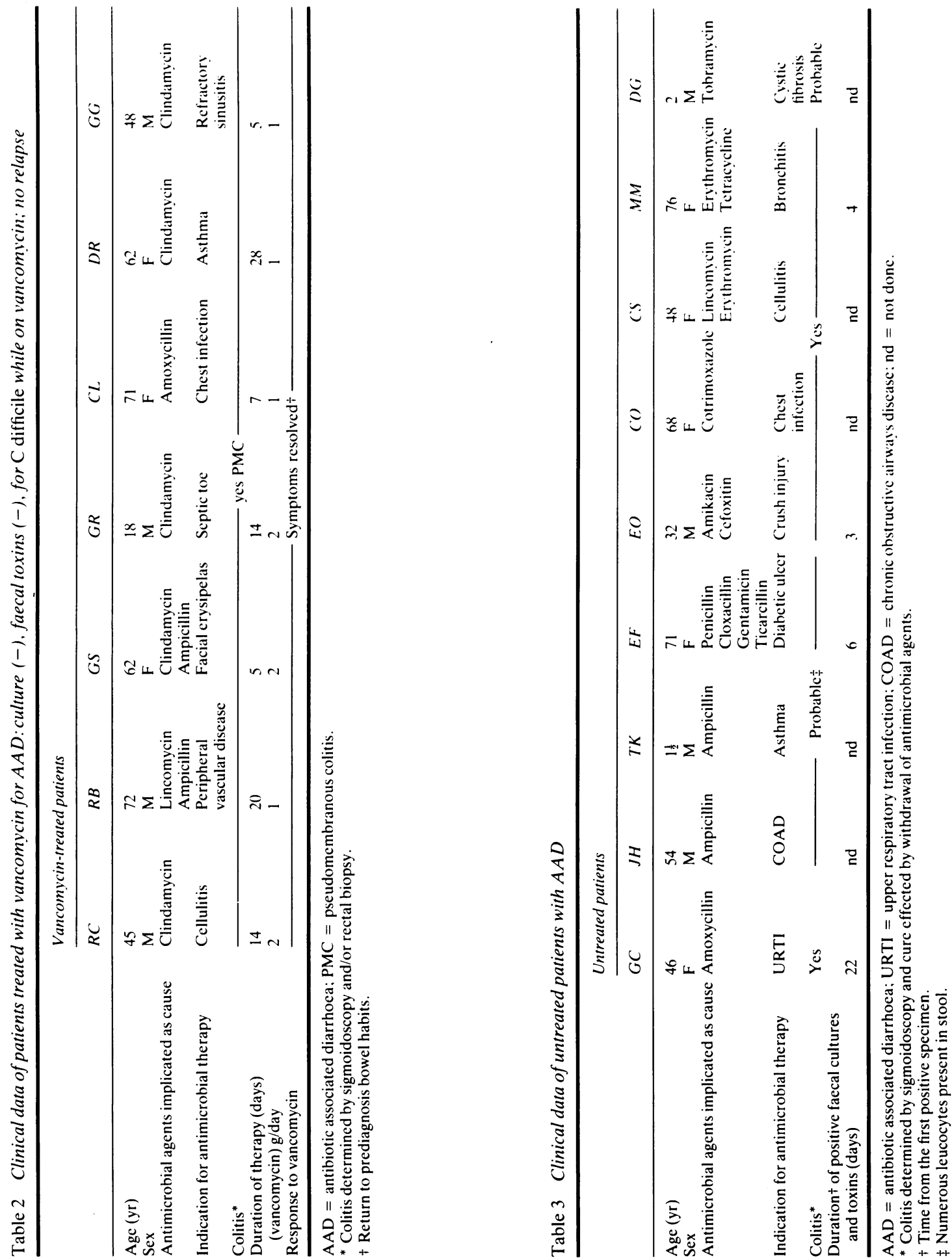
specimens were difficult to obtain from this group after diarrhoea terminated. In this group four patients were co-operative and the persistence of $C$ difficile as well as the cytotoxin in these patients ranged from three days to 22 days.

$C$ difficile was readily isolated from the selective agar media (CCFA. CDA) and recovered from the broth for all the initial specimens submitted. In five out of eight patients. however, who had relapses isolation of $C$ difficile during treatment was only successful from the $p$ cresol broth. There was an abrupt end to the successful cultivation of the organism from the seven patients successfully treated with vancomycin, often after the first day of treatment. In two out of seven patients (RB and CL) the last specimen from which $C$ difficile was isolated was via the $\mathrm{p}$ cresol broth. This also occurred with two patients (EO and $\mathrm{MM}$ ) who were not treated. Resistance to vancomycin (MIC $>2 \mu \mathrm{g} / \mathrm{ml}$ ) was never encountered with any of the strains isolated and all the strains were toxigenic.

\section{Discussion}

These results confirm the high incidence of relapse of antibiotic associated colitis after treatment with vancomycin. For the patients treated with vancomycin in this report, relapse was not apparently related to sex, age, cytotoxin concentration, severity of the disease, or previous exposure to any particular antibiotic. Patients treated with vancomycin with no relapse, however, were exposed to fewer antibiotics than the patients who relapsed. Four of the eight patients who relapsed received three antibiotics and one received two. Their course of vancomycin was at a slightly higher dose $(1.9 \mathrm{~g} v \mathrm{~s}$ $1.3 \mathrm{~g} /$ day) and for a shorter period ( 9.8 days vs 13.3 days) than patients treated with vancomycin but who did not relapse.

Relapse in patients treated with vancomycin was associated with the continued presence of $C$ difficile, in the absence of detectable cytotoxin, in the stool. This suggests that the relapses observed in this report may be the result of the endogenous presence of the organism despite the high levels of vancomycin shown to exist in the stool by the treatment protocols used. ${ }^{23}$ All strains of $C$ difficile isolated were sensitive to vancomycin although in vitro sensitivity to a given antibiotic does not guarantee its success in eradicating $C$ difficile. There have been several reports of antibiotic associated colitis caused by isolates highly susceptible to the antibiotic implicated as the cause. ${ }^{21} 22$ Further work is required to understand the mechanism by which $C$ difficile is able to survive antibiotic therapy in vivo. Relapse was not encountered in any of the patients treated by withdrawal of antibiotics; none of these patients required antibiotics in the ensuing six months.

The nature of the changes in susceptibility of the bowel to colonisation by $C$ difficile are not known. Clearly the source of $C$ difficile in an individual who develops pseudomembranous colitis is critical to an understanding of the epidemiology of this disease in the community as a whole. If the source of $C$ difficile is initially endogenous, antibiotic therapy may allow the organism to proliferate although antibiotic associated colitis is not simply due to overgrowth of toxin producing $C$ difficile. ${ }^{2.3}$ Alternatively, it may be that the major source of $C$ difficile is exogenous as is suggested by several reports of clusters of pseudomembranous colitis, and also our own report of the epidemiology of the disease in an intensive care unit. ${ }^{1+2425}$ Reinfection from exogenous sources may be potential cause of relapse.

The bacteriological data presented here would suggest that one of the reasons for relapse is the persistence of the organism presumably as spores within the affected colon. The fact that faecal toxin disappears quickly implies that the number of vegetative forms of $C$ difficile are rapidly reduced by therapy with vancomycin or vancomycin inhibits toxin production in vivo. On cessation of vancomycin therapy reversion to vegetative forms with recrudesence of the disease may potentially occur if the appropriate conditions are present. Little is understood of the interactions between normal faecal flora and $C$ difficile but the observation that certain strains of Staphylococcus, Pseudomonas, Bacteroides, and Lactobacillus inhibit the growth of $C$ difficile and that the growth of certain strains of the genera Peptococcus, Peptostreptococcus, and Bacteroides were inhibited by $C$ difficile suggests a fine balance exists between the various organisms colonising the normal large bowel. ${ }^{12}$ Presumably if this balance is not restored before vegetation of spores occurs, a relapse of the illness may be possible.

Thus failure to demonstrate a cytotoxin may be because of its non-production by $C$ difficile in the spore form, its low concentration by reduced numbers of the vegetative form, or loss of the ability of $C$ difficile to produce toxin. Some evidence for all these mechanisms was observed in the eight patients who relapsed. $C$ difficile was isolated during vancomycin treatment from the broth in only five patients, suggesting the presence of spores. Two patients (CD and $\mathrm{RF}$ ) had severely reduced normal faecal flora but continued to excrete the organism in large numbers during treatment although a cytotoxin was not detected. These strains which grew on all the media used were subsequently shown 
to produce cytotoxin after growth in cooked meat medium for 48 hours. The reason why these strains did not produce cytotoxin during vancomycin therapy is not known. Patient AM had normal faecal flora present and there were only a few colonies of $C$ difficile on the CCFA media (a technique capable of detecting as few as $100 \mathrm{cfu}$ of $C$ difficile/g of stool). ${ }^{17}$ The absence of cytotoxin from the specimens obtained from this patient during vancomycin therapy may have been the result of cytotoxin levels below detectable limits by the tissue culture method. There is some evidence that the initial dilution necessary in the tissue culture method may prevent the detection of low levels of the toxin. ${ }^{26}$

The results reported in this series of patients with antibiotic associated colitis may be of value in prognosis and treatment. Whereas mild cases of antibiotic associated colitis respond well to the withdrawal of antibiotic therapy a significant number of the more serious cases treated with vancomycin may require more prolonged and intensive treatment to prevent relapse. This is presumably because $C$ difficile is able to re-establish infection after vancomycin therapy which is ineffectual when $C$ difficile is present in spores. Cultural methods that include selective enrichment techniques have the advantage of detecting spores or low numbers of the organism. We have evidence that the preliminary enrichment in non-selective broth before plating to CCFA is more sensitive than direct plating alone for the recovery of $C$ difficile from stool (unpublished observations). The presence of $C$ difficile in the stool of patients being treated with vancomycin for antibiotic associated colitis may potentially identify those patients predisposed to relapse. Finally, if the rapid reestablishment of the normal bowel flora is essential for the prevention of relapse then a more specific antibiotic for $C$ difficile should be used. As no such antibiotic is available at the present time, lower or intermittent doses of vancomycin may have less effect on the normal bowel flora.

This study was supported by a grant from the Department of Veterans' Affairs of the Australian Government. We wish to thank Mrs W Gemmell and Miss $\mathrm{J}$ Thursby for secretarial assistance. We are grateful to the various local clinicians who referred patients and provided specimens for this study. Mr R Grice, State Virology Laboratory, Brisbane, kindly performed the toxin assays.

\section{References}

1 Modigliani R, Delchien JC. Vancomycin for antibiotic- induced colitis. Lancet 1978; 1: 97-8.

2 Tedesco F, Markham R, Gurwith M, Christic D. Bartlett JG. Oral vancomycin for antibiotic-associated pscudomembranous colitis. Lancet 1978: 2: 226-8.

3 Keighley MRB, Burdon DW, Arabi Y el al. Randomised controlled trial of vancomycin for pseudomembranous colitis and postoperative diarrhoea. $\mathrm{Br}$ Med J 1978; 2: 1667-9.

4 George WL, Volpicelli NA, Stiner OB et al. Relapse of pseudomembranous colitis after vancomycin therapy. $N$ Engl J Med 1979; 301: 414-5.

5 Roberts RK, Seneviratne E. Vancomycin therapy (Costridium difficile. Med J Aust 1980; 2: 98.

6 Bartlett JG, Tedesco FJ, Shull S, Lowe B, Chang T. Symptomatic relapse after oral vancomycin therapy of antibiotic-associated pseudomembranous colitis. Gastroenterology 1980; 78: 431-4.

7 George WL, Kirby BD, Sutter VL et al. Antimicrobial susceptibility of Clostridium difficile. In: Schlessinger D, ed. Microbiology - 1979. Washington, DC: American Society for Microbiology, 1979: 267-71.

8 Trinh Dinh H, Kernbaum S, Frottier J. Treatment of antibiotic-induced colitis by metronidazole. Lancet 1978; 1: 338-9.

9 Kceutzer EW, Milligan FD. Treatment of antibioticassociated pseudomembranous colitis with cholestyramine resin. Johns Hopkins Med J 1978; 143: $67-72$.

10 Onderdonk AB, Cisneros R, Bartlett JG. Study of Clostridium difficile gnotobiotic mice. Infect Immunol 1980; 28(1): 277-82.

11 Onderdonk AB, Lowe BR, Bartlett JG. Effect of environmental stress on Clostridium difficile toxin levels during continuous cultivation. Appl Environ Microbiol 1979; 38: 637-41.

12 Rolfe RD, Finegold SM. Inhibitory interactions between normal fecal flora and Clostridium difficile. Am J Clin Nutr 1980; 33: 2539.

13 Mulligan ME, George WL, Rolfe RD, Finegold SM. Epidemiological aspects of Clostridium difficileinduced diarrhoea and colitis. Am J Clin Nutr 1980; 33: 2533-8.

14 Walters BAJ, Stafford R, Roberts RK, Seneviratne E. Contamination and crossinfection with Clostridium difficile in an intensive care unit. Aust NZ J Med 1982; 12: (3) 255-8.

$15 \mathrm{Kim} \mathrm{KH}$, Fekety $\mathrm{R}$, Batts $\mathrm{DH}$ et al. Isolation of Clostridium difficile from the environment and contacts of patients with antibiotic-associated colitis. $J$ Infect Dis 1981; 143: 42-50.

16 Chang T, Lavermann M, Bartlett JG. Cytotoxicity assay in antibiotic associated colitis. J Infect Dis 1979; 140: $765-70$.

17 George WL, Sutter VL, Citron D, Finegold SM. A selective and differential medium for the isolation of Clostridium difficile. J Clin Microbiol 1979; 9: 214-9.

18 Hafiz S, Oakley CL. Clostridium difficile: isolation and characteristics. J Med Microbiol 1976; 9: 129-36.

19 Holdeman CK, Cato EP, Moore WEC. Anaerobic laboratory manual, 4th ed. Blocksburg, Virginia: Virginia Polytechnic Institute and State University. 1977. 
20) Wilkins TD. Thiel T. Modified broth-disc method for testing the antibiotic susceptibility of anaerobic bacteria. Antimicrob Agents (hemother 1973: 3: 350-6.

21 Saginur R. Hawiev CR. Bartlett JR. Colitis associated with metronidazole therapy. J Infect Dis 1980): 141: 772-4.

22 George WL. Sutter VL. Fincgold SM. Toxigenicity and antimicrobial susceptibility of Clostridium difficile as a cause of antimicrohial agent-associated colitis. Curr Microbiol 1978: 1: 5.5-8.
23 Onderdonk AB. Bartlett JG. The biological and clinical significance of Clostridium difficile. Crit Rev Clin Lab Sci 1981: 13: (3): 161-72.

24 Keighley MRB. Burdon DW. Mogg RH et al. Pseudomembranous colitis. Lancet 1979: 1: 559-60.

25 Kabins SA. Spira TJ. Outbreak of clindamycinassociated colitis. Ann Intern Med 1975; 83: 830-1.

26 Ryan RW. Kwasnik I, Tilton RC. Rapid detection of Clostridium difficile toxin in human faeces. J Clin Microbiol 1980; 12: 776-9. 